Research Article; Received: February 23, 2021; Accepted: July 7, 2021

\title{
Q-MEROMORPHIC CLOSE-TO-CONVEX FUNCTIONS RELATED WITH JANOWSKI FUNCTION
}

\author{
F. Müge SAKAR ${ }^{1}$, Syed Ghoos Ali SHAH ${ }^{2}$, Saqib HUSSAIN ${ }^{2}$, \\ Akhter RASHEED ${ }^{2}$, and Muhammad NAEEM ${ }^{3}$ \\ ${ }^{1}$ Department of Management, Dicle University, Diyarbakır, TURKEY \\ ${ }^{2}$ Department of Mathematics, Comsats University Islamabad, Abbottabad Campus, PAKISTAN \\ ${ }^{3}$ Department of Mathematics and Statistic, International Islamic University Islamabad, \\ PAKISTAN
}

ABstract. In the present paper, we introduce and explore certain new classes of meromorphic functions related to closed-to-convexity and $q$-calculus. Such results as coefficient estimates, grow the property and partial sums are derived. It is important to mention that our results are generalization of number of existing results in literature.

\section{INTRODUCTION}

Let $\sum_{1}$ denote the class of meromorphic functions of the form:

$$
f(\omega)=\frac{1}{\omega}+\sum_{t=1}^{\infty} a_{t} \omega^{t}
$$

which are analytic in the punctured open unit disc $U^{*}=\{\omega: \omega \in \mathbb{C}$ and $0<\{\omega\}<1\}=U \backslash\{0\}$, where $U=U^{*} \cup\{0\}$.

In Geometric Function Theory, several subclasses of the meromorphic functions have already been examined and investigated through many perceptions, see( 9,10 , 12 18 $18,21,22]$ ). Ismail et al. 8 , were the first to use the q-derivative operator $\Delta_{q}$ in order to study a certain $q$-analogue of the class $T^{*}$ of starlike functions in $U$. Certain basic properties of the $q$-close-to-convex functions were studied by Raghavendar and Swaminathan [28], Aral et al. [2] successfully studied the applications of the

2020 Mathematics Subject Classification. Primary 30C45, Secondary 30C5.

Keywords. Meromorphic starlike function, Janowski functions, $q$-derivative.

@ mugesakar@hotmail.com-Corresponding author; alishahsyedghoos@gmail.com; saqib_math@yahoo.com; akhter_rasheed77@yahoo.com; naeem.phdma75@iiu.edu.pk

(D) 0000-0002-3884-3957; 0000-0003-4283-8058; 0000-0002-8174-8795; 0000-0003-3810-5214 0000-0002-9069-3095. 
$q$-calculus in operator theory. In fact, they found significant application of the $q$-calculus mainly in the Geometric Function Theory. Moreover, the generalized $q$-hypergeometric function was first introduced by Srivastava [26], see also( $1,3,5$, $6,14,16,20]$ ).

A function $f \in \sum_{1}$ is said to be meromorphic starlike of order $\alpha$ defined as:

$$
f \in \sum^{M S}(\alpha) \Leftrightarrow \operatorname{Re}\left(\frac{\omega f^{\prime}(\omega)}{f(\omega)}\right)<-\alpha \quad\left(0 \leq \alpha<1 ; \omega \in U^{*}\right) .
$$

A related class of meromorphic convex function $\sum^{M C}(\alpha)$ is defined as:

$$
f \in \sum^{M C}(\alpha) \Leftrightarrow \operatorname{Re}\left(1+\frac{\omega f^{\prime \prime}(\omega)}{f^{\prime}(\omega)}\right)<-\alpha \quad\left(\omega \in U^{*}\right)
$$

By $\sum^{M K}(\alpha)$, we mean $f \in \sum_{1}$ and the class of all close-to-convex functions which satisfies the condition

$$
\operatorname{Re}\left(\frac{\omega f^{\prime}(\omega)}{g(\omega)}\right)<-\alpha, \quad \text { where } g \in \sum^{M S}(\alpha) .
$$

The study of operators plays main role in the theory of geometric functions. Many differential and integral operators can be written in terms of convolution of certain holomorphic functions.

For $g(\omega)=\frac{1}{\omega}+\sum_{t=0}^{\infty} b_{t} \omega^{t} \in \sum_{1}$ and $f$ given in 1 . The Convolution (Hadamard product) is denoted by $f * g$ and defined as:

$$
(f * g)(\omega)=\frac{1}{\omega}+\sum_{t=0}^{\infty} a_{t} b_{t} \omega^{t}=(g * f)(\omega) .
$$

A function $h$ analytic in $U$ and of the form

$$
h(\omega)=1+\sum_{t=1}^{\infty} r_{t} \omega^{t}
$$

A given function $\Psi$ with $\Psi(0)=1$ is said to belong to the class $S^{*}[A, B]$ if and only if

$$
\Psi(\omega) \prec \frac{1+A \omega}{1+B \omega} \quad(-1 \leq B<A \leq 1) .
$$

This class was presented and studied by Janowski [11]. By taking $A=1$ and $B=-1$, we obtain the class $P$ of functions with a positive real part. It is important to mention that $\Psi(\omega) \in S^{*}[A, B]$ if and only if there exists $r \in P$ such that

$$
\Psi(\omega)=\frac{(A+1) R(\omega)-(A-1)}{(B+1) R(\omega)-(B-1)} \quad(-1 \leq B<A \leq 1) .
$$


Motivated by the works of Srivastava et al. see( $7,17,19,23,25,27])$ also see( 4 13 $15,24,29$ ). In this paper, we shall consider new subfamilies of $q$ meromorphic close-to-convex functions with respect to Janowski functions.

Throughout in this paper, we assume

$$
\begin{aligned}
0 \leq \eta<1,-1 \leq B<A \leq 1,0 \leq q<1, \omega \in U^{*}, f, g \in \sum_{1}, \\
\Lambda(t, \eta, A, q)=\left[\left|b_{t}\right|\left|\left(2[t]_{q} \eta+2(1-\eta)+\eta(A+1)\right)-(A+1)(1-\eta)\right|\right], \\
\Lambda(t, B, q)=[t]_{q}(2+B+1),
\end{aligned}
$$

and

$$
\gamma(\eta, A, B, q)=|(B+1)+(A+1) \eta-(A+1)(1-\eta) q|+2(1-\eta)(1-q),
$$

unless otherwise mentioned.

Definition 1. (see [9] and [10]) The q-derivative (q-difference) $\Delta_{q}$ of a function $f$ is defined in a given subset of $\mathbb{C}$ by

$$
\left(\Delta_{q} f\right)(\omega)= \begin{cases}\frac{f(\omega)-f(q \omega)}{(1-q) \omega} & (\omega \neq 0), \\ f^{\prime}(0) & (\omega=0),\end{cases}
$$

where $0<q<1$. This implies the following.

$$
\lim _{q \rightarrow 1^{-}}\left(\Delta_{q} f\right)(\omega)=\lim _{q \rightarrow 1^{-}} \frac{f(\omega)-f(q \omega)}{(1-q) \omega}=f^{\prime}(\omega),
$$

provided that $f^{\prime}(0)$ exists.

The function $\Delta_{q} f$ has Maclaurin's series representation

$$
\left(\Delta_{q} f\right)(\omega)=\sum_{t=0}^{\infty}[t]_{q} a_{t} \omega^{t-1},
$$

where $q \in(0,1)$ and define the q-number $[\gamma]_{q}$ by

$$
[\gamma]_{q}=\left\{\begin{array}{lr}
\frac{1-q^{\gamma}}{1-q} & (\gamma \in \mathbb{C}), \\
\sum_{k=0}^{t-1} q^{\gamma}=1+q^{2}+q^{3}+\ldots+q^{t-1} & (t \in \mathbb{N}) .
\end{array}\right.
$$

For more details about q-derivatives, we refer the reader to (see [6]).

Definition 2. For $f \in \sum_{1}$, let the q-derivative operator (q-difference operator) be defined by

$$
\left(\Delta_{q} f\right)(\omega)=\frac{f(q \omega)-f(\omega)}{(q-1) \omega}=-\frac{1}{q \omega^{2}}+\sum_{t=0}^{\infty}[t]_{q} a_{t} \omega^{t-1} \quad\left(\omega \in U^{*}\right) .
$$

Similarly

$$
\left(\Delta_{q} g\right)(\omega)=\frac{g(q \omega)-g(\omega)}{(q-1) \omega}=-\frac{1}{q \omega^{2}}+\sum_{t=0}^{\infty}[t]_{q} b_{t} \omega^{t-1} \quad\left(\omega \in U^{*}\right) .
$$


Definition 3. A function $f \in \sum_{1}$ is said to belong to the class $f \in T_{(q, \eta)}^{*}[A, B]$ if and only if

$$
\left|\frac{(B-1)\left(\frac{-\omega \Delta_{q} f(\omega)}{(1-\eta) g(\omega)+\eta \omega \Delta_{q} g(\omega)}\right)-(A-1)}{(B+1)\left(\frac{-\omega \Delta_{q} f(\omega)}{(1-\eta) g(\omega)+\eta \omega \Delta_{q} g(\omega)}\right)-(A+1)}-\frac{1}{1-q}\right|<\frac{1}{1-q} .
$$

Where $g \in \sum^{M S}(\alpha)$, It is easily observed that

$$
\lim _{q \rightarrow 1^{-}} T_{(q, 0)}^{*}[A, B]=S_{q}^{M K}[A, B],
$$

secondly we have

$$
\lim _{q \rightarrow 1^{-}} T_{(q, 0)}^{*}[1,-1]=S_{q}^{M K},
$$

where $S_{q}^{M K}[A, B]$ is the well-known function of meromorphic close-to-convex function.

\section{Main Results}

\subsection{Coefficient estimates.}

Theorem 1. A function $f \in \sum_{1}$ of the form given by (1) is in the class $T_{(q, \eta)}^{*}[A, B]$ if it satisfies the following condition.

$$
\sum_{t=1}^{\infty}\left(\Lambda(t, B, q)\left|a_{t}\right| q+(t, \eta, A, q)\left|b_{t}\right| q\right) \leq \gamma(\eta, A, B, q)
$$

where

$$
\begin{gathered}
\Lambda(t, B, q)=[t]_{q}(2+B+1) \\
\Lambda(t, \eta, A, q)=\left[\left|b_{t}\right|\left|\left(2[t]_{q} \eta+2(1-\eta)+\eta(A+1)\right)-(A+1)(1-\eta)\right|\right]
\end{gathered}
$$

and

$$
\gamma(\eta, A, B, q)=|(B+1)+(A+1) \eta-(A+1)(1-\eta) q|+2(1-\eta)(1-q) .
$$

Proof. Assuming that (8) holds, it suffices to show that

$$
\left|\frac{(B-1)\left(\frac{-\omega \Delta_{q} f(\omega)}{(1-\eta) g(\omega)+\eta \omega \Delta_{q} g(\omega)}\right)-(A-1)}{(B+1)\left(\frac{-\omega \Delta_{q} f(\omega)}{(1-\eta) g(\omega)+\eta \omega \Delta_{q} g(\omega)}\right)-(A+1)}-\frac{1}{1-q}\right|<\frac{1}{1-q} .
$$

Consider we have

$$
\left|\frac{(B-1)\left(\frac{-\omega \Delta_{q} f(\omega)}{(1-\eta) g(\omega)+\eta \omega \Delta_{q} g(\omega)}\right)-(A-1)}{(B+1)\left(\frac{-\omega \Delta_{q} f(\omega)}{(1-\eta) g(\omega)+\eta \omega \Delta_{q} g(\omega)}\right)-(A+1)}-\frac{1}{1-q}\right|
$$

which implies

$$
=\left|\frac{-(B-1) \omega \Delta_{q} f(\omega)-(A-1)\left[(1-\eta) g(\omega)+\eta \omega \Delta_{q} g(\omega)\right]}{-(B+1) \omega \Delta_{q} f(\omega)-(A+1)\left[(1-\eta) g(\omega)+\eta \omega \Delta_{q} g(\omega)\right]}-1\right|+\frac{q}{1-q}
$$


Thus

$$
2\left|\frac{\omega \Delta_{q} f(\omega)+(1-\eta) g(\omega)+\eta \omega \Delta_{q} g(\omega)}{-(B+1) \omega \Delta_{q} f(\omega)-(A+1)\left[(1-\eta) g(\omega)+\eta \omega \Delta_{q} g(\omega)\right]}\right|+\frac{q}{1-q}
$$

Using (1), (6) and (7) in above equation.

$$
\left|\begin{array}{c}
2(1-\eta)(q-1) \\
+2 \sum_{t=1}^{\infty}\left[[t]_{q}\left(a_{t}+\eta b_{t}\right)+(1-\eta) b_{t}\right] q \omega^{t+1} \\
(B+1)+(A+1) \eta-(A+1)(1-\eta) q \\
-\sum_{t=1}^{\infty}\left[[t]_{q}\left((B+1) a_{t}+\eta(A+1) b_{t}\right)-(A+1)(1-\eta) b_{t}\right] q \omega^{t+1}
\end{array}\right| \leq 1
$$

we get

$$
\begin{aligned}
& \sum_{t=1}^{\infty}[t]_{q}\left|a_{t}\right|(2+B+1) q+\sum_{t=1}^{\infty}\left[\left|b_{t}\right|\left|\left(2[t]_{q} \eta+2(1-\eta)+\eta(A+1)\right)-(A+1)(1-\eta)\right|\right] q \\
& \leq|(B+1)+(A+1) \eta-(A+1)(1-\eta) q|+2(1-\eta)(1-q) .
\end{aligned}
$$

The last expression become

$$
\sum_{t=1}^{\infty} \Lambda(t, B, q)\left|a_{t}\right| q+\sum_{t=1}^{\infty} \Lambda(t, \eta, A, q)\left|b_{t}\right| q \leq \gamma(\eta, A, B, q) .
$$

This complete the proof of Theorem 2.1.

Corollary 1. If a function $f \in \sum_{1}$ of the form given by (1) is in the class $T_{(q, \eta)}^{*}[A, B]$, then

$$
\left|a_{t}\right| \leq \frac{\gamma(\eta, A, B, q)}{\Lambda(t, B, q)}-\frac{\Lambda(t, \eta, A, q)}{\Lambda(t, B, q)}\left|b_{t}\right| \quad(t \in N),
$$

with equality for each $t$, we define the function of the form

$$
f_{t}(\omega)=\frac{1}{\omega}+\left(\frac{\gamma(\eta, A, B, q)}{\Lambda(t, B, q)}-\frac{\Lambda(t, \eta, A, q)}{\Lambda(t, B, q)}\left|b_{t}\right|\right) \omega^{t},
$$

where $\Lambda(t, B, q), \Lambda(t, \eta, A, q)$ and $\gamma(\eta, A, B, q)$ are given by (9), (10) and (11) respectively.

\subsection{Distortion inequalities.}

Theorem 2. If $f \in T_{(q, \eta)}^{*}[A, B]$, then

$$
\begin{aligned}
& \frac{1}{r}-\left(\frac{\gamma(\eta, A, B, q)}{\Lambda(1, B, q)}-\frac{\Lambda(t, \eta, A, q)}{\Lambda(1, B, q)}\left|b_{t}\right|\right) r \leq|f(\omega)| \\
\leq & \frac{1}{r}+\left(\frac{\gamma(\eta, A, B, q)}{\Lambda(1, B, q)}-\frac{\Lambda(t, \eta, A, q)}{\Lambda(1, B, q)}\left|b_{t}\right|\right) r(|\omega|=r),
\end{aligned}
$$

where equality holds for the function

$$
f(\omega)=\frac{1}{\omega}+\left(\frac{\gamma(\eta, A, B, q)}{\Lambda(1, B, q)}-\frac{\Lambda(1, \eta, A, q)}{\Lambda(1, B, q)}\left|b_{t}\right|\right) \omega .
$$


Proof. Let $f \in T_{(q, \eta)}^{*}[A, B]$. Then in view of Theorem (2.1), we have

$$
\Lambda(1, B, q) \sum_{t=1}^{\infty}\left|a_{t}\right| \leq \sum_{t=1}^{\infty} \Lambda(t, B, q)\left|a_{t}\right| \leq \gamma(\eta, A, B, q)-\sum_{t=1}^{\infty} \Lambda(1, \eta, A, q)\left|b_{t}\right|,
$$

which yields

$$
|f(\omega)| \leq \frac{1}{r}+\sum_{t=1}^{\infty}\left|a_{t}\right| r^{t} \leq \frac{1}{r}+r \sum_{t=1}^{\infty}\left|a_{t}\right| \leq \frac{1}{r}+\left(\frac{\gamma(\eta, A, B, q)}{\Lambda(1, B, q)}-\frac{\Lambda(1, \eta, A, q)}{\Lambda(1, B, q)}\left|b_{t}\right|\right) r .
$$

Similarly, we have

$$
|f(\omega)| \geq \frac{1}{r}-\sum_{t=1}^{\infty}\left|a_{t}\right| r^{t} \geq \frac{1}{r}-r \sum_{t=1}^{\infty}\left|a_{t}\right| \geq \frac{1}{r}-\left(\frac{\gamma(\eta, A, B, q)}{\Lambda(1, B, q)}-\frac{\Lambda(1, \eta, A, q)}{\Lambda(1, B, q)}\left|b_{t}\right|\right) r
$$

which is required.

Theorem 3. If $f \in T_{(q, \eta)}^{*}[A, B]$, then

$$
\begin{aligned}
& \frac{1}{r^{2}}-2\left(\frac{\gamma(\eta, A, B, q)}{\Lambda(1, B, q)}-\frac{\Lambda(t, \eta, A, q)}{\Lambda(1, B, q)}\left|b_{t}\right|\right) \\
& \leq|f(\omega)| \leq \frac{1}{r^{2}}+2\left(\frac{\gamma(\eta, A, B, q)}{\Lambda(1, B, q)}-\frac{\Lambda(t, \eta, A, q)}{\Lambda(1, B, q)}\left|b_{t}\right|\right) \quad(|\omega|=r),
\end{aligned}
$$

where equality holds for the function

$$
f(\omega)=\frac{1}{\omega}+\left(\frac{\gamma(\eta, A, B, q)}{\Lambda(1, B, q)}-\frac{\Lambda(1, \eta, A, q)}{\Lambda(1, B, q)}\left|b_{t}\right|\right) \omega .
$$

Proof. Let $f \in T_{(q, \eta)}^{*}[A, B]$. Then in view of theorem (2.1), we have

$$
\Lambda(1, B, q) \sum_{t=1}^{\infty}\left|a_{t}\right| \leq \sum_{t=1}^{\infty} \Lambda(t, B, q)\left|a_{t}\right| \leq \gamma(\eta, A, B, q)-\sum_{t=1}^{\infty} \Lambda(1, \eta, A, q)\left|b_{t}\right| .
$$

Differentiate (14) and 15), we get

$$
\left|f^{\prime}(\omega)\right| \leq-\frac{1}{r^{2}}+\sum^{\infty} t\left|a_{t}\right| r^{t-1} \leq-\frac{1}{r^{2}}+\sum_{t=1}^{\infty}\left|a_{t}\right| \leq-\frac{1}{r^{2}}+\left(\frac{\gamma(\eta, A, B, q)}{\Lambda(1, B, q)}-\frac{\Lambda(1, \eta, A, q)}{\Lambda(1, B, q)}\left|b_{t}\right|\right) .
$$

Similarly, we have

$$
\left|f^{\prime}(\omega)\right| \geq-\frac{1}{r^{2}}-\sum^{\infty} t\left|a_{t}\right| r^{t-1} \geq-\frac{1}{r^{2}}-\sum_{t=1}^{\infty}\left|a_{t}\right| \geq-\frac{1}{r^{2}}-\left(\frac{\gamma(\eta, A, B, q)}{\Lambda(1, B, q)}-\frac{\Lambda(1, \eta, A, q)}{\Lambda(1, B, q)}\left|b_{t}\right|\right) .
$$

Comparing (16) and (17).

We have thus completed the proof of Theorem 2.4. 


\subsection{Partial sums.}

In this section, we examine the ratio of a function of the form (1) to its sequence of partial sums

$$
f_{t}(\omega)=\frac{1}{\omega}+\sum_{t=1}^{k} a_{t} \omega^{t}
$$

when the coefficients of $f$ are sufficiently small to satisfy condition (8). We will determine sharp lower bounds for

$\operatorname{Re}\left(\frac{f(\omega)}{f_{\nu}(\omega)}\right), \quad \operatorname{Re}\left(\frac{f_{\nu}(\omega)}{f(\omega)}\right), \quad \operatorname{Re}\left(\frac{\left(\Delta_{q} f\right)(\omega)}{\left(\Delta_{q} f_{\nu}\right)(\omega)}\right) \quad$ and $\quad \operatorname{Re}\left(\frac{\left(\Delta_{q} f_{\nu}\right)(\omega)}{\left(\Delta_{q} f\right)(\omega)}\right)$.

Theorem 4. If $f$ of the form (1) satisfies condition (8), then

$$
\operatorname{Re}\left(\frac{f(\omega)}{f_{\nu}(\omega)}\right) \geq 1-\frac{1}{\kappa_{\nu+1}} \quad\left(\omega \in U^{*}\right)
$$

and

where

$$
\operatorname{Re}\left(\frac{f_{\nu}(\omega)}{f(\omega)}\right) \geq \frac{\kappa_{\nu+1}}{1+\kappa_{\nu+1}} \quad\left(\omega \in U^{*}\right)
$$

$$
\kappa_{\nu}=\frac{\gamma(\eta, A, B, q)}{\Lambda(t, B, q)}-\frac{\Lambda(t, \eta, A, q)}{\Lambda(t, B, q)}\left|b_{t}\right| .
$$

Proof. In order to prove inequality (18), we set

$$
\begin{aligned}
\kappa_{\nu+1}\left[\frac{f(\omega)}{f_{\nu}(\omega)}-\left(1-\frac{1}{\kappa_{\nu+1}}\right)\right] & =\frac{1+\sum_{t=1}^{\nu} a_{t} \omega^{t-1}+\kappa_{\nu+1} \sum_{t=\nu+1}^{\infty} a_{t} \omega^{t+1}}{1+\sum_{t=1}^{\nu} a_{t} \omega^{t+1}} \\
& =\frac{1+h_{1}(\omega)}{1+h_{2}(\omega)} .
\end{aligned}
$$

Let

$$
\frac{1+h_{1}(\omega)}{1+h_{2}(\omega)}=\frac{1+g(\omega)}{1-g(\omega)} .
$$

Finally, to prove the inequality in $(18)$, we get

$$
\sum_{t=1}^{\nu}\left(1-\kappa_{\nu+1}\right)\left|a_{t}\right|+\sum_{t=\nu+1}^{\infty}\left(\kappa_{\nu+1}-\kappa_{t}\right)\left|a_{t}\right| \geq 0 .
$$

The proof of inequality in 18 is now completed.

Similarly, we set

$$
1+\kappa_{\nu}\left[\frac{f_{\nu}(\omega)}{f(\omega)}-\left(\frac{\kappa_{\nu+1}}{1+\kappa_{\nu+1}}\right)\right]=\frac{1+\sum_{t=1}^{\nu} a_{t} \omega^{t-1}-\kappa_{\nu+1} \sum_{t=\nu+1}^{\infty} a_{t} \omega^{t-1}}{1+\sum_{t=1}^{\nu} a_{t} \omega^{t-1}}
$$




$$
=\frac{1+g(\omega)}{1-g(\omega)} .
$$

We have completed the proof of $(19)$, which complete the proof of Theorem 2.5 .

Theorem 5. If $f$ of the form (1) satisfies condition (8), then

$$
\operatorname{Re}\left(\frac{\left(\Delta_{q} f\right)(\omega)}{\left(\Delta_{q} f_{\nu}\right)(\omega)}\right) \geq 1-\frac{[\nu+1]_{q}}{\kappa_{\nu+1}} \quad\left(\omega \in U^{*}\right),
$$

and

$$
\operatorname{Re}\left(\frac{\left(\Delta_{q} f_{\nu}\right)(\omega)}{\left(\Delta_{q} f\right)(\omega)}\right) \geq \frac{\kappa_{\nu+1}}{\kappa_{\nu+1}+[\nu+1]_{q}} \quad\left(\omega \in U^{*}\right)
$$

where $\kappa_{\nu}$ is given by $(20)$.

The proof of Theorem 2.6, is similar to that of Theorem 2.5.

\subsection{Radius of starlikeness.}

In the next theorem we find the radius of $q$-starlikeness for the class $T_{(q, \eta)}^{*}[A, B]$.

Theorem 6. Let the function $f$ given by (1) be in the class $T_{(q, \eta)}^{*}[A, B]$. Then $f$ is meromorphic starlike of order $\alpha$ in $|\omega| \leq r$, where

$$
r=i n f_{t \geq 1}\left[\frac{(1-\alpha) \Lambda(t, B, q)}{(n+2-\alpha)\left[\gamma(\eta, A, B, q)-\Lambda(t, \eta, A, q)\left|b_{t}\right|\right]}\right]^{\frac{1}{t+1}},
$$

Proof. In order to prove above result, we must show that

$$
\left|\frac{f^{\prime}(\omega)}{f(\omega)}+1\right| \leq 1-\alpha \quad(0 \leq \alpha<1) \quad \text { and } \quad|\omega| \leq r_{1},
$$

we have

$$
\begin{gathered}
\left|\frac{f^{\prime}(\omega)}{f(\omega)}+1\right|=\frac{\sum_{t=1}^{\infty}(t+1) a_{t} \omega^{t}}{\frac{1}{\omega}+\sum_{t=1}^{\infty} a_{t} \omega^{t}} . \\
\leq \frac{\sum_{t=1}^{\infty}(t+1)\left|a_{t}\right||\omega|^{t+1}}{1-\sum_{t=1}^{\infty}\left|a_{t}\right||\omega|^{t+1}}
\end{gathered}
$$

Since the appropriate condition for a function $f$ to be in the class $\sum^{M S}(\alpha)$ is given by

$$
\sum_{t=1}^{\infty}(t+\alpha)\left|a_{t}\right|<1-\alpha \quad\left(0 \leq \alpha<1 ; \omega \in U^{*}\right) .
$$

Hence (23) holds true if

$$
\sum_{t=1}^{\infty}(t+1)\left|a_{t}\right||\omega|^{t+1} \leq(1-\alpha) \quad\left(1-\sum_{t=1}^{\infty}\left|a_{t}\right||\omega|^{t+1}\right) .
$$


The inequality in 25 can be written as:

$$
\sum_{t=1}^{\infty}\left(\frac{t+2-\alpha}{1-\alpha}\right)\left|a_{t}\right||\omega|^{t+1} \leq 1
$$

With the aid of (8), inequality (26) is true if

$$
\left(\frac{t+2-\alpha}{1-\alpha}\right)|\omega|^{t+1} \leq \frac{\Lambda(t, B, q)}{\gamma(\eta, A, B, q)-\Lambda(t, \eta, A, q)\left|b_{t}\right|} .
$$

Solving (27) for $|\omega|$, we have

$$
|\omega|=\left[\frac{(1-\alpha) \Lambda(t, B, q)}{(n+2-\alpha)\left[\gamma(\eta, A, B, q)-\Lambda(t, \eta, A, q)\left|b_{t}\right|\right]}\right]^{\frac{1}{t+1}} .
$$

In view of 28) the proof of our theorem is now completed.

Definition 4. A function $f \in \sum_{1}$ is said to belong to the class $f \in T_{(q, \eta, 1)}^{*}[A, B]$ if and only if

$$
\operatorname{Re}\left(\frac{(B-1) \frac{\omega \Delta_{q} f(\omega)}{(1-\eta) g(\omega)+\eta \omega \Delta_{q} g(\omega)}-(A-1)}{(B+1) \frac{\omega \Delta_{q} f(\omega)}{(1-\eta) g(\omega)+\eta \omega \Delta_{q} g(\omega)}-(A+1)}\right) \geq 0 .
$$

We call $T_{(q, \eta, 1)}^{*}[A, B]$ the class of $q$ close-to-convex function of Type 1 related with the Janowski functions.

Definition 5. A function $f \in \sum_{1}$ is said to belong to the class $f \in T_{(q, \eta, 2)}^{*}[A, B]$ if and only if

$$
\left|\frac{(B-1) \frac{\omega \Delta_{q} f(\omega)}{(1-\eta) g(\omega)+\eta \omega \Delta_{q} g(\omega)}-(A-1)}{(B+1) \frac{\omega \Delta_{q} f(\omega)}{(1-\eta) g(\omega)+\eta \omega \Delta_{q} g(\omega)}-(A+1)}-\frac{1}{1-q}\right|<\frac{1}{1-q} .
$$

We call $T_{(q, \eta, 2)}^{*}[A, B]$ the class of $q$ close-to-convex function of Type 2 related with the Janowski functions.

Definition 6. A function $f \in \sum_{1}$ is said to belong to the class $f \in T_{(q, \eta, 3)}^{*}[A, B]$ if and only if

$$
\left|\frac{(B-1) \frac{\omega \Delta_{q} f(\omega)}{(1-\eta) g(\omega)+\eta \omega \Delta_{q} g(\omega)}-(A-1)}{(B+1) \frac{\omega \Delta_{q} f(\omega)}{(1-\eta) g(\omega)+\eta \omega \Delta_{q} g(\omega)}-(A+1)}-1\right|<1 .
$$

We call $T_{(q, \eta, 3)}^{*}[A, B]$ the class of $q$ close-to-convex function of Type 3 related with the Janowski functions.

\section{For Special Cases.}

(1) For $\eta=0$ and $g(\omega)=f(\omega)$ then $T_{(q, 0,)}^{*}[A, B], T_{(q, 0,1)}^{*}[A, B], T_{(q, 0,2)}^{*}[A, B]$ and $T_{(q, 0,3)}^{*}[A, B]$ classes reduced to $S_{q}^{*}[A, B], S_{(q, 1)}^{*}[A, B], S_{(q, 2)}^{*}[A, B]$ and $S_{(q, 3)}^{*}[A, B]$ studied by Srivastava et al 17,27 . 
(2) For $\eta=0, g(\omega)=f(\omega), A=1-2 \alpha$ and $B=-1$ in $T_{(q, 0,)}^{*}[A, B]$, $T_{(q, 0,1)}^{*}[A, B], T_{(q, 0,2)}^{*}[A, B]$ and $T_{(q, 0,3)}^{*}[A, B]$ we get the classes $S_{q}^{*}, S_{(q, 1)}^{*}(\alpha), S_{(q, 2)}^{*}(\alpha)$ and $S_{(q, 3)}^{*}(\alpha)$, which was introduced and studied by Wongsaijai and Sukantamala (see 30$]$ ).

\subsection{Main Results and Their Demonstration.}

We first derive the presence results for the succeeding generalized $q$-starlike functions:

$$
T_{(q, \eta, 1)}^{*}[A, B], \quad T_{(q, \eta, 2)}^{*}[A, B] \quad \text { and } \quad T_{(q, \eta, 3)}^{*}[A, B],
$$

which are associated with the Janowski functions.

Theorem 7. If $-1 \leq B<A<1$, then

$$
T_{(q, \eta, 3)}^{*}[A, B] \subset T_{(q, \eta, 2)}^{*}[A, B] \subset T_{(q, \eta, 1)}^{*}[A, B] .
$$

Proof. First of all, we suppose that $f \in T_{(q . \eta, 3)}^{*}[A, B]$. Then, by Definition 2.10, we have

so that

$$
\left|\frac{(B-1) \frac{\omega \Delta_{q} f(\omega)}{(1-\eta) g(\omega)+\eta \omega \Delta_{q} g(\omega)}-(A-1)}{(B+1) \frac{\omega \Delta_{q} f(\omega)}{(1-\eta) g(\omega)+\eta \omega \Delta_{q} g(\omega)}-(A+1)}-1\right|<1,
$$

$$
\left|\frac{(B-1) \frac{\omega \Delta_{q} f(\omega)}{(1-\eta) g(\omega)+\eta \omega \Delta_{q} g(\omega)}-(A-1)}{(B+1) \frac{\omega D_{q} f(\omega)}{(1-\eta) g(\omega)+\eta \omega \Delta_{q} g(\omega)}-(A+1)}-1\right|+\frac{q}{1-q}<1+\frac{q}{1-q} .
$$

By using the triangle inequality and equation 29 , we find that

$$
\left|\frac{(B-1) \frac{\omega \Delta_{q} f(\omega)}{(1-\eta) g(\omega)+\eta \omega \Delta_{q} g(\omega)}-(A-1)}{(B+1) \frac{\omega \Delta_{q} f(\omega)}{(1-\eta) g(\omega)+\eta \omega \Delta_{q} g(\omega)}-(A+1)}-\frac{1}{1-q}\right|<\frac{1}{1-q} .
$$

The last expression in 30 now implies that $f \in T_{(q, \eta, 2)}^{*}[A, B]$, that is, that

$$
T_{(q, 3)}^{*}[A, B] \subset T_{(q, 2)}^{*}[A, B] .
$$

Next, we let $f \in T_{(q, \eta, 2)}^{*}[A, B]$, so that

$f \in T_{(q, \eta, 2)}^{*}[A, B] \Longleftrightarrow\left|\frac{(B-1) \frac{\omega \Delta_{q} f(\omega)}{(1-\eta) g(\omega)+\eta \omega \Delta_{q} g(\omega)}-(A-1)}{(B+1) \frac{\omega \Delta_{q} f(\omega)}{(1-\eta) g(\omega)+\eta \omega \Delta_{q} g(\omega)}-(A+1)}-\frac{1}{1-q}\right|<\frac{1}{1-q}$.

As we know

$$
\begin{gathered}
\frac{1}{1-q}>\left|\frac{(B-1) \frac{\omega \Delta_{q} f(\omega)}{(1-\eta) g(\omega)+\eta \omega \Delta_{q} g(\omega)}-(A-1)}{(B+1) \frac{\omega \Delta_{q} f(\omega)}{(1-\eta) g(\omega)+\eta \omega \Delta_{q} g(\omega)}-(A+1)}-\frac{1}{1-q}\right| \\
\quad=\left|\frac{1}{1-q}-\frac{(B-1) \frac{\omega \Delta_{q} f(\omega)}{(1-\eta) g(\omega)+\eta \omega \Delta_{q} g(\omega)}-(A-1)}{(B+1) \frac{\omega D_{q} f(\omega)}{(1-\eta) g(\omega)+\eta \omega \Delta_{q} g(\omega)}-(A+1)}\right|,
\end{gathered}
$$


we have

$$
\operatorname{Re}\left(\frac{(B-1) \frac{\omega \Delta_{q} f(\omega)}{(1-\eta) g(\omega)+\eta \omega \Delta_{q} g(\omega)}-(A-1)}{(B+1) \frac{\omega \Delta_{q} f(\omega)}{(1-\eta) g(\omega)+\eta \omega D_{q} g(\omega)}-(A+1)}\right)>0 \quad\left(\omega \in U^{*}\right) .
$$

This last equation now shows that $f \in T_{(q, \eta, 1)}^{*}[A, B]$, that is, that

$$
T_{(q \cdot \eta, 2)}^{*}[A, B] \subset T_{(q \cdot \eta, 1)}^{*}[A, B] .
$$

We have thus completed the proof of Theorem 2.11.

Theorem 8. Let $f \in \sum_{1}$, then $f \in T_{(q, \eta, 2)}^{*}[A, B]$ if and only if

$$
\left|\frac{f(q \omega)}{(1-\eta) g(\omega)+\eta g(q \omega)}-\frac{\varkappa}{(B-1) q+B+3}\right| \leqq \frac{(A+1)(1-q)}{(B-1) q+B+3},
$$

where

$$
\varkappa=(A-1) q^{2}+(B-A+2) q+B+1 .
$$

Proof. Let

$$
\frac{\omega \Delta_{q} f(\omega)}{(1-\eta) g(\omega)+\eta \omega \Delta_{q} g(\omega)}=\left(\frac{1}{1-q}\right)\left(1-\frac{f(q \omega)}{(1-\eta) g(\omega)+\eta g(q \omega)}\right) .
$$

Using Definition 2.9 of the class $T_{(q, \eta, 2)}^{*}[A, B]$ associated with the Janowski functions.

$$
\left|\frac{(B-1)\left(\frac{1}{1-q}\right)\left(1-\frac{f(q \omega)}{(1-\eta) g(\omega)+\eta g(q \omega)}\right)-(A-1)}{(B+1)\left(\frac{1}{1-q}\right)\left(1-\frac{f(q \omega)}{(1-\eta) g(\omega)+\eta g(q \omega)}\right)-(A+1)}-\frac{1}{1-q}\right|<\frac{1}{1-q}
$$

We have thus completed the proof of Theorem 2.12 .

Corollary 2. It is worth mentioning that the classes

$$
T_{(q, \eta, 1)}^{*}[A, B], \quad T_{(q, \eta, 2)}^{*}[A, B] \text { and } T_{(q, \eta, 3)}^{*}[A, B] .
$$

of the generalized $q$ closed-to-convex functions of Type1, Type 2, and Type3, respectively, satisfy the following properties:

$$
\cap_{q \in(0,1)} T_{(q, \eta, 1)}^{*}[A, B]=\cap_{q \in(0,1)} T_{(q, \eta, 2)}^{*}[A, B]=T^{*}[A, B] .
$$

and

$$
\cap_{q \in(0,1)} T_{(q, \eta, 1)}^{*}[A, B]=\bigcap_{q \in(0,1)} T_{(q, \eta, 3)}^{*}[A, B] \subset T^{*}[A, B] .
$$

Let $L$ be a subset of $\sum_{1}$ consisting of functions with a negative coefficient, that is,

$$
f(\omega)=\frac{1}{\omega}-\sum_{t=1}^{\infty}\left|a_{t}\right| \omega^{t} \quad\left(a_{t} \geq 0\right) .
$$

We also let

$$
L T_{(q, \eta, t)}^{*}[A, B]=T_{(q, \eta, t)}^{*}[A, B] \cap L \quad(t=1,2,3) .
$$


Theorem 9. For $-1 \leq B<A<1$, then

$$
L T_{(q, \eta, 1)}^{*}[A, B]=L T_{(q, \eta, 2)}^{*}[A, B]=L T_{(q, \eta, 3)}^{*}[A, B] .
$$

Proof. In view of Theorem 2.11, it is sufficient here to show that

$$
L T_{(q, \eta, 1)}^{*}[A, B] \subset L T_{(q, \eta, 3)}^{*}[A, B] .
$$

Indeed, if we assume that $f \in L T_{(q \cdot \eta, 1)}^{*}[A, B]$, then we have

$$
\operatorname{Re}\left(\frac{(B-1) \frac{\omega \Delta_{q} f(\omega)}{(1-\eta) g(\omega)+\eta \omega \Delta_{q} g(\omega)}-(A-1)}{(B+1) \frac{\omega \Delta_{q} f(\omega)}{(1-\eta) g(\omega)+\eta \omega \Delta_{q} g(\omega)}-(A+1)}\right) \geq 0,
$$

so that

$$
\operatorname{Re}\left(\frac{(B-1) \frac{\omega \Delta_{q} f(\omega)}{(1-\eta) g(\omega)+\eta \omega \Delta_{q} g(\omega)}-(A-1)}{(B+1) \frac{\omega \Delta_{q} f(\omega)}{(1-\eta) g(\omega)+\eta \omega \Delta_{q} g(\omega)}-(A+1)}-1\right) \geq-1 .
$$

After a simple calculation, we thus find that

$$
2\left|\frac{-\omega \Delta_{q} f(\omega)+(1-\eta) g(\omega)+\eta \omega \Delta_{q} g(\omega)}{(B+1) \omega \Delta_{q} f(\omega)-(A+1)\left[(1-\eta) g(\omega)+\eta \omega \Delta_{q} g(\omega)\right]}\right| \geq-1
$$

Using (1), (6) and (7) in above equation.

$$
\left|\begin{array}{c}
2(2 \eta-1)-2(1-\eta) q+ \\
2 \sum_{t=1}^{\infty}\left[[t]_{q}\left(a_{t}-\eta b_{t}\right)-(1-\eta) b_{t}\right] q \omega^{t+1} \\
-(B+1)+(A+1) \eta-(A+1)(1-\eta) q \\
+\sum_{t=1}^{\infty}\left[[t]_{q}\left((B+1) a_{t}-\eta(A+1) b_{t}\right)-(A+1)(1-\eta) b_{t}\right] q \omega^{t+1}
\end{array}\right|<1
$$

This implies we get

$$
\begin{aligned}
& \sum_{t=1}^{\infty}\left|a_{t}\right|[t]_{q}(2-(B+1)) q+\sum_{t=1}^{\infty}\left[\begin{array}{c}
-2[t]_{q} \eta+(A+1) \eta \\
+(A+1)(1-\eta)-2(1-\eta)
\end{array}\right]\left|b_{t}\right| q \\
& \leq|(B+1)-(A+1) \eta-(A+1)(1-\eta) q|+2(1-\eta) q-2(2 \eta-1),
\end{aligned}
$$

which satisfies $T_{(q, \eta, 3)}^{*}[A, B]$. By Definition 2.10, the proof of Theorem 2.14 is completed.

\section{Conclusion}

In our current investigation, we have presented and studied thoroughly some new subclasses of $q$ meromorphic close-to-convex functions, which is connected with the Janowski functions. Then we discussed some interesting properties and characteristics of these new subclasses, including distortion theorem, radius problem and partial sum. Some special cases have been discussed as applications of our main results. The technique and ideas of this paper may stimulate further research in this dynamic field. 
Author Contribution Statements All authors contributed equally to design and implementation of the research. They jointly analyzed the results and wrote the manuscriprt. They read and approved the final manuscript.

Declaration of Competing Interests The authors declare that they have no competing interest.

Acknowledgments The authors are thankful to the editor and referee(s) for their valuable comments and suggestions.

\section{REFERENCES}

[1] Aouf, M. K., Silverman, H., Partial sums of certain meromorphic $p$-valent functions, J. Inequal. Pure Appl. Math., 7(4), Article 119, (2006).

[2] Aral, A., Gupta, V., Agarwal, R. P., Applications of $q$-Calculus in Operator Theory, Springer, New York, USA, 2013.

[3] Cho, N. E., Owa, S., Sufficient conditions for meromorphic starlikeness and close to-convexity of order $\alpha$, Int. J. Math. Sci., 26(5) (2001), 317-319. DOI:10.1155/S0161171201004550

[4] Clunie, J., On meromorphic schlicht functions, J. London Math. Soc., 34 (1959), 215-216.

[5] Darus, M., Hussain, S., Raza, M., Sokol, J., On a subclass of starlike functions, Results in Mathematics, 73 (2018), 1-12.

[6] Gasper, G., Rahman, M., Basic Hypergeometric Series, Cambridge University Press Cambridge, 1990.

[7] Hussain, S., Srivastava, H. M., Raziq, A., Raza, M., The Fekete-Szego functional for subclass of analytic functions associated with quasi-subordination, Carpathian J. Math., 34 (2018), 103-113.

[8] Ismail, M. E. H., Merkes, E., Styer, D., A generalization of starlike functions, Complex Var. Theory Appl., 14 (1990), 77-84.

[9] Jackson, F. H., On q-definite integrals, Quart. J. Pure Appl. Math., 41 (1910), 193-203.

[10] Jackson, F. H., q-difference equations, Am. J. Math., 32 (1910), 305-314.

[11] Janowski, W., Some extrenal problems for certain families of analytic functions, Ann. Pol. Math., 28 (1973), 297-326.

[12] Khan, B., Liu, Z. G., Srivastava,H. M., Khan, N., Darus, M., Tahir, M., A study of some families of multivalent $q$-starlike functions involving higher-order $q$-derivatives, Mathematics, 8 (2020), Article ID 1470, 1-12. DOI:10.3390/math8091470

[13] Khan, N., Shafiq, M., Darus, M., Khan, B., Ahmad, Q. Z., it Upper bound of the third Hankel determinant for a subclass of $q$-starlike functions associated with Lemniscate of Bernoulli, $J$. Math. Inequal., 1 (2020), 51-63. DOI:10.7153/jmi-2020-14-05

[14] Miller, J. E., Convex meromrphic mapping and related functions, Proc. Amer. Math. Soc., 25 (1970), 220-228.

[15] Miller, S. S., Mocanu, P. T., Differential subordination and univalent functions, Mich. Math. J., 28 (1918), 157-171.

[16] Miller, S. S., Mocanu, P. T., Differential Subordination Theory and Applications Series on Monographs and Textbooks in Pure and Applied Mathematics, Vol. 225, Marcel Dekker Inc., New York, 2000.

[17] Mahmood, S., Ahmed, q. Z., Srivastava, H. M., Khan, N., Khan, B., Tahir, M., A certain subclass of meromorphically q-starlike functions associated with the Janowski function, J.Inequal. Appl. Math., 2019(88) (2019), 1-11. https://doi.org/10.1186/s13660-019-2020-z 
[18] Naeem, M., Hussain, S., Sakar, F. M., Mahmood T., Rasheed, A., Subclasses of uniformly convex and starlike functions associated with Bessel functions, Turkish Journal of Mathematics, 2019(43) (2019), 2433-2443. DOI:10.3906/mat-1905-27

[19] Owa, s., Srivastava, H. M., Current Topics in Analytic Function Theory, World Scientific, Singapore, 1992. https://doi.org/10.1142/1628

[20] Pommerenke, C., On meromorphic starlike functions. Pac. J. Math., 13 (1963), 221-235.

[21] Rasheed, A., Hussain, S., Shah, S. G. A., Darus, M., Lodhi, S., Majorization problem for two subclasses of meromorphic functions associated with a convolution operator, AIMS Mathematics, 5(5) (2020), 5157-5170. doi: 10.3934/math.2020331

[22] Srivastava, H. M., Operators of basic (or q-) calculus and fractional q-calculus and their applications in geometric function theory of complex analysis, Iran. J. Sci. Technol. Trans. A: Sci., 44(2020) (2020), 327-344.

[23] Srivastava, H. M., Tahir, M., Khan, B., Ahmad, Q. Z., Khan, N., Some general families of qstarlike functions associated with the Janowski functions, Filomat, 33(9) (2019), 2613-2626. https://doi.org/10.2298/FIL1909613S

[24] Shah, S. G. A., Hussain, S., Rasheed, A., Shareef, Z., Darus, M., Application of quasisubordination to certain classes of meromorphic functions, Journal of Function Spaces, Vol. (2020), Article ID 4581926, 8 pages. https://doi.org/10.1155/2020/4581926

[25] Srivastava, H. M., Hossen, H. M., Aouf, M, K., A unified presentation of some classes of meromorphically multivalent functions, Comput. Math. Appl., 38(11-12), (1999), 63-70.

[26] Srivastava, H. M., Bansal, D., Close-to-convexity of a certain family of $q$-Mittag-Leffler functions, J. Nonlinear Var. Anal., 1 (2017), 61-69.

[27] Srivastava, H. M., Tahir, M., Khan, B., Ahmed, Q. Z., Khan, N., Some general classes of $q$-starlike functions associated with the Janowski function, Symmetry, 11 (2019), Article ID 292, 1-14. DOI:10.3390/sym11020292

[28] Swaminathan, K., Raghavendar, A close-to-convexi of basic hypergeometric functions using their Taylor coefficients, J. Math. Appl., 35 (2012), 111-125.

[29] Shah, S. G. A., Noor, S., Darus, M., W. Ul Haq, S. Hussain, On meromorphic functions defined by a new class of liu-srivastava integral operator, International Journal of Analysis and Applications, 18(6) (2020), 1056-1065.

[30] Wongsaijai, B., Sukantamala, N., Certain properties of some families of generalized starlike functions with respect to q-calculus. Abstr. Appl. Anal., (2016), 1-8. https://doi.org/10.1155/2016/6180140 